Received: 2015.10 .02 Accepted: 2016.01.19 Published: 2016.05 .17

Authors' Contribution: Study Design A Data Collection B Statistical Analysis C Data Interpretation D Manuscript Preparation E Literature Search F Funds Collection $G$

\title{
Binding of ATGs to Endothelial Cells In Vivo
}

\author{
ABCDEFG 1 Andres Beiras-Fernandez \\ DEF 1,2 Astrid Hernandez-Sierra \\ CD 3 Uwe Schulz \\ BCD 4 Manfred Richter \\ ACDE 5 Eckart Thein \\ D 1 Anton Moritz \\ ABCD 1 Isabella Werner
}

\author{
1 Department of Thoracic and Cardiovascular Surgery, JW Goethe University, \\ Frankfurt, Germany \\ 2 Department of Nephrology, Autonomous University of Bucaramanga (UNAB), \\ Bucaramanga, Colombia \\ 3 Department of Cardiac Surgery, Das Herz- und Diabeteszentrum (HDZ), \\ Bad Oyenhausen, Germany \\ 4 Department of Cardiac Surgery, Kerkhoff-Klinik, Bad Nauheim, Germany \\ 5 Institute for Surgical Research, Ludwig-Maximilian-University, Munich, Germany
}

Corresponding Author: Source of support:
Andres Beiras-Fernandez, e-mail: Andres.Beiras@kgu.de Departmental sources

Background: Polyclonal anti-thymocyte globulins (ATGs) are immunosuppressive drugs widely used in induction of immunosuppression and treatment of acute rejection after solid organ transplantation. We have previously demonstrated that ATGs bind to endothelial cells in vitro, and are able to modulate ECs. The aim of this study was to investigate the binding of ATGs to endothelial cells under in vivo conditions.

Material/Methods: Muscle biopsies from extremities of cynomolgus monkeys were obtained after ischemia/reperfusion at $4^{\circ} \mathrm{C}$. ATGs (Thymoglobulin, Sanofi-Aventis, France; $1 \mathrm{mg} / \mathrm{kg}$ ) were added to the blood $30 \mathrm{~min}$ prior to the reperfusion. Biopsies $(n=10)$ of patients undergoing heart transplantation and preoperatively treated with ATGs (Thymoglobulin, Sanofi-Aventis, France; $1.5 \mathrm{mg} / \mathrm{kg}$ ) as induction therapy were also analyzed 6 hours and 7 days after induction. Binding of ATGs to ECs was analyzed with an anti-rabbit lgG antibody by means of immunohistochemistry.

Results: Binding of ATGs to endothelial cells could be demonstrated in vivo in our animal experiments 4 hours after reperfusion, as well as in the clinical biopsies 6 hours after induction of immunosuppression in heart transplant patients, showing a preferred localization in post-capillary veins. No expression of ATGs on the endothelial surface could be observed after 7 days, suggesting that ATGs may be washed out from the endothelial surface in a time-dependent manner.

Conclusions: Our results show that ATGs are able to bind to endothelial cells in an experimental model and in clinical practice, supporting preconditioning strategies with ATGs in solid organ transplantation.

MeSH Keywords: Endothelial Cells • Heart Transplantation • Immunosuppressive Agents

Full-text PDF: http://www.annalsoftransplantation.com/abstract/index/idArt/896158

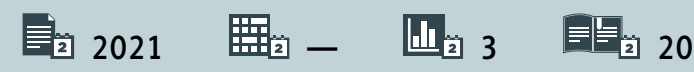




\section{Background}

Polyclonal anti-thymocyte globulins (ATGs) are immunosuppressive drugs widely used in induction of immunosuppression and treatment of acute rejection after solid organ transplantation [1]. The main mechanism of action has been extensively reported, and is based on the depletion of activated T-cells through complement activation and induction of apoptosis through CD95 [2,3]. However, ATGs present other mechanisms of action not related to T-cell depletion, allowing different immunosuppressive strategies [3,4], including modulation of endothelial cells and regulation of dendritic cells and B-cells [4-6].

Induction of immunosuppression has been historically related to low rates occurrence of rejection episodes and better graft function [7]. Recently, Djamali et al. [8] demonstrated reduction of the incidence of donor-specific antibodies (DSA) in patients receiving induction with rabbit anti-thymocyte globulin (Thymoglobulin, Sanofi-Aventis, Boston, USA). In their report, the reduction of the incidence of DSA was also related to better long-term outcome. As possible mechanisms of action of ATGs in this context, they proposed a depletion of naive B-cells, as demonstrated by Popov et al., or a possible reduction of antigen presenting cells (APC) through T-regs [9].

We have previously demonstrated that ATGs bind to endothelial cells in vitro, and are able to modulate ECs $[5,10]$. As DSAs also originate from endothelial cells, we propose the hypothesis that the polyclonal antibodies bind to endothelial cells and that this "coating" of endothelial cells prevents the expression of endothelial antigens to circulating recipient cells. The aim of our study was to demonstrate, as "proof of concept", that ATGs bind to endothelial cells under in vivo conditions.

\section{Material and Methods}

\section{Tissue samples}

\section{Animal biopsies}

Biopsies of muscle tissue of cynomolgus monkeys $(n=10)$ were obtained after ischemia-reperfusion injury and treatment with $1 \mathrm{mg} / \mathrm{kg}$ ATG ( $\mathrm{n}=5$; Thymoglobulin, Sanofi-Aventis, Germany), or without ATG (Control; $n=5$ ). The Bavarian government and the local ethics committee (N: AZ-211-2531-33/2000) approved this study. A complete description of the methodical setup, including surgical procedures, has been described elsewhere [11] Briefly, 10 cynomolgus monkeys (Macaca fasciculata) were anaesthetized and provided with central lines. The axillary and inguinal vessels were isolated from the main circulation and cannulated to allow connection to an extracorporeal perfusion system, as described before [12]. After rinsing the animal's

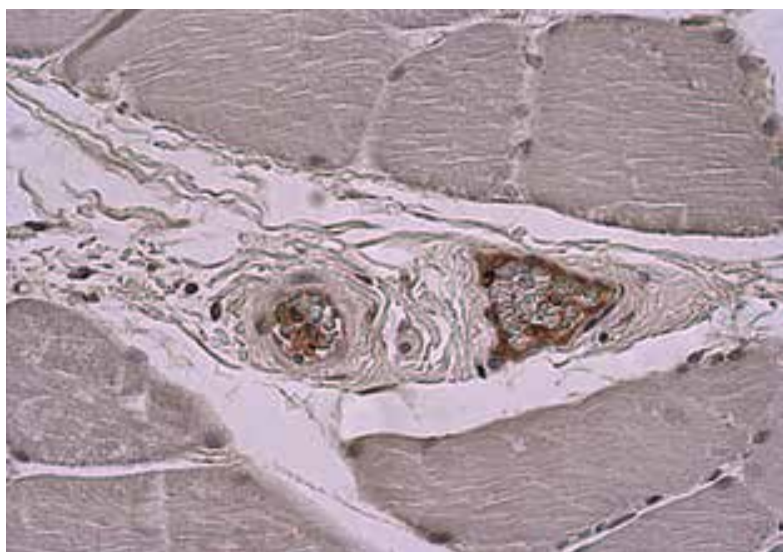

Figure 1. Representative microphotography of positive staining of endothelial cells with anti-rabbit IgG, demonstrating binding of ATGs to the ECs in our experimental primate model $(\times 40)$.

blood from the extremity by flushing with Ringer's lactate solution at $4^{\circ} \mathrm{C}$ for $60 \mathrm{~min}$, reperfusion with human blood treated with $1 \mathrm{mg} / \mathrm{kg}$ ATG or untreated blood was initiated. ATGs were added to the human blood $30 \mathrm{~min}$ before the experiments. Reperfusion lasted for $240 \mathrm{~min}$. The animals were euthanized after the experiments. Biopsies of muscle and connective tissue were obtained directly after the experiments, stored in formalin for $48 \mathrm{~h}$, and then embedded in paraffin to be fixed for immunohistochemical analysis (Figure 1).

\section{Human biopsies}

Cardiac biopsies $(n=10)$ were obtained from patients undergoing heart transplantation after institutional review board approval (AZ 168/12). Induction of immunosuppression with $1.5 \mathrm{mg} / \mathrm{kg}$ of rATG (Thymoglobulin; Sanofi-Aventis, Germany) was made preoperatively in all cases during induction of anaesthesia after insertion of an arterial line to control blood pressure. Preoperative induction was performed to delay administration of anticalcineurinics. Biopsies were obtained from the diseased heart after explantation (right atrium; left/right ventricle). At the end of the implantation and after weaning of CPB, 1 tissue sample of the right atrium was obtained from the cannulation site of the donor heart (Figure 2). Biopsies from patients undergoing ventricular assist device (VAD) implantation were used as controls to demonstrate the specificity of the technique (Figure 3$)$. Biopsies ( $n=4)$ taken to rule out acute rejection were made as early as 7 days after transplantation, and only per protocol or clinical suspicion. The biopsies were stored in $4 \%$ formalin for 48-72 $\mathrm{h}$ and embedded in paraffin for immunohistochemical and histological studies.

\section{Histology and immunohistochemistry}

Tissue sections (5- $\mu \mathrm{m}$-thick) of animal and human origin were mounted on Histobond adhesion microslides, and then were 

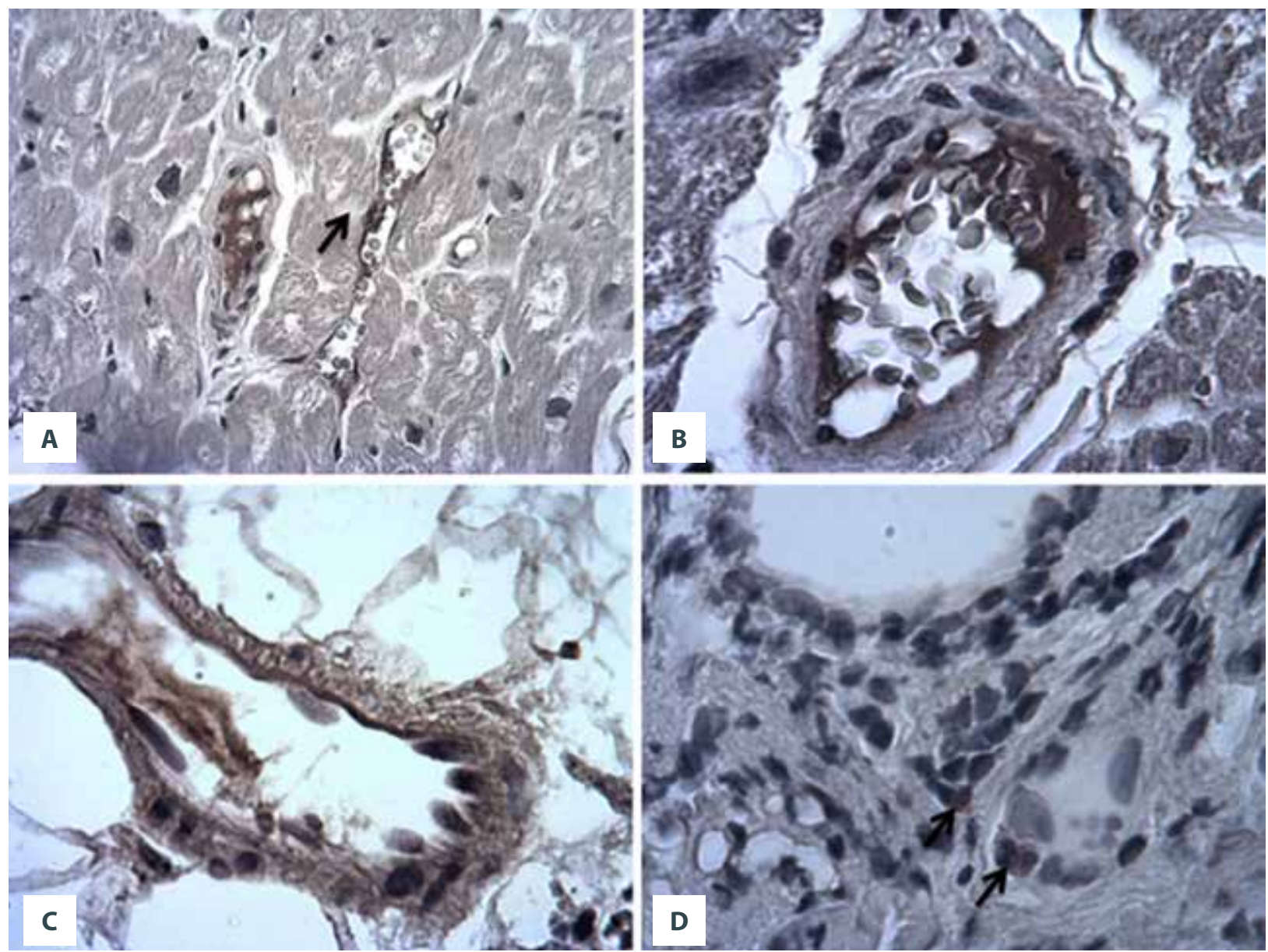

Figure 2. Positive reaction after staining with anti-rabbit IgG upon cardiac biopsies after heart transplantation. (A) Explanted heart 4 $\mathrm{h}$ after induction with ATG ( $\times 20)$; (B) Explanted heart $4 \mathrm{~h}$ after induction with ATG $(\times 40)$; (C) Donor heart $6 \mathrm{~h}$ after induction with ATG (x40); (D) Donor heart 7 days after induction with ATG $(\times 40)$.
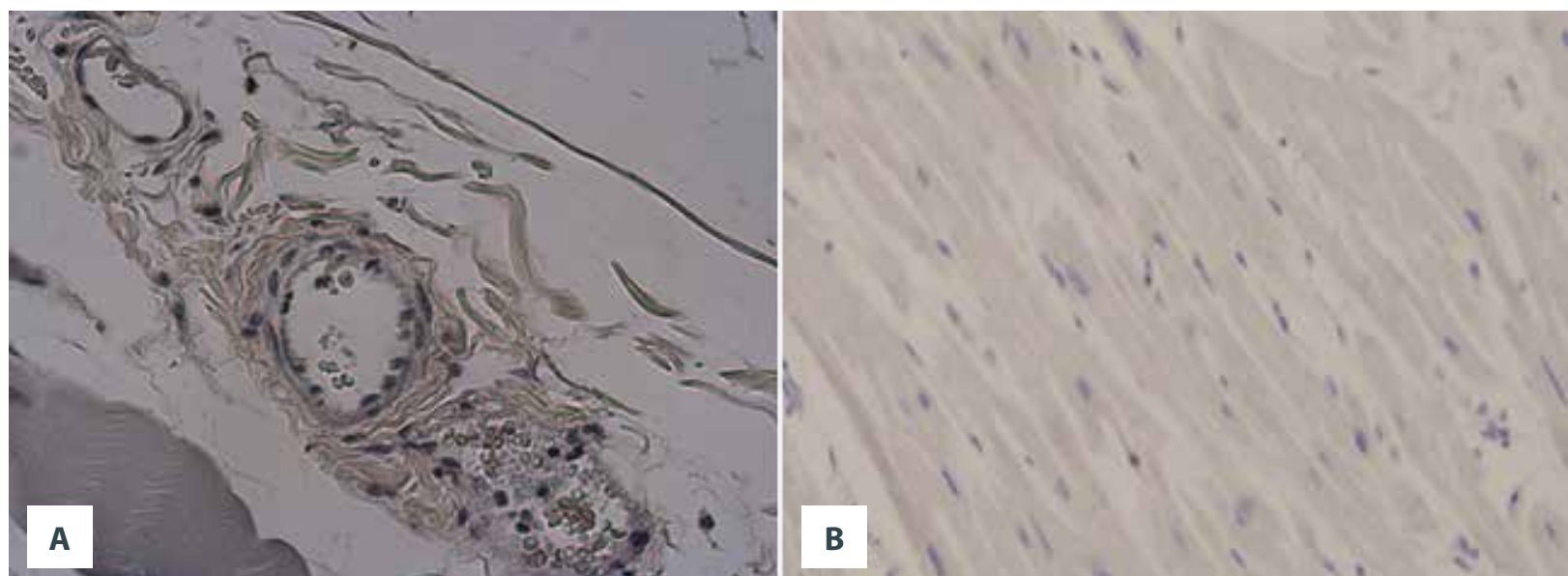

Figure 3. Negative reaction after staining with anti-rabbit IgG in animal and human cardiac biopsies. (A) Muscle after no treatment with ATG (×20); (B) Heart after no induction with ATG $(\times 20)$.

dewaxed and rehydrated. Hematoxylin/Eosin (H/E) staining was performed in 1 of every 10 slices to check for adequate orientation of the preparation. H/E and Masson's trichrome techniques were performed to localize the most important types of cells, vessels, and muscle fibers, as well as to differentiate muscle fibers, cells nuclei, fibrin, and collagen (data not shown). Binding 
of ATGs to endothelial cells and other cell types was assessed with an anti-rabbit IgG antibody (Abcam, Cambridge, UK). Sections from animal (after the experiments) and human tissue ( $6 \mathrm{~h}$ and 7 days after induction with ATG) were evaluated.

For immunohistochemistry, antigen retrieval was carried out by heating in a microwave oven for $10 \mathrm{~min}$ at $750 \mathrm{~W}$ or by $20 \mathrm{~min}$ of pressure-cooking in $10 \mathrm{mM}$ sodium citrate buffer $(\mathrm{pH} 6.0)$. Sections were consecutively incubated in: (1) anti-Rabbit IgG polyclonal antibody (Abcam, Cambridge; UK) at a dilution of 1:100 for $2 \mathrm{~h}$ according to manufacturer`s protocol; (2) 3\% hydrogen peroxide (Merck, Darmstadt, Germany) to block endogenous peroxidase for $10 \mathrm{~min}$; (3) visualization reagent (dextran polymer-conjugated with horseradish peroxidase and affinityisolated goat anti-rabbit immunoglobulins) (Envision ${ }^{\odot}$, Dako, Glostrup, Denmark) for $30 \mathrm{~min}$. In each step, sections were washed twice for $5 \mathrm{~min}$ with TBS (50 mM Tris buffer, $\mathrm{pH}$ 7.6, containing $150 \mathrm{mM} \mathrm{NaCl}$ ). All dilutions were made in TBS except for the primary antibody (step 1), in which ChemMate ${ }^{\odot}$ antibody diluent (Dako) was used. All sections were counterstained with hematoxylin for $1 \mathrm{~min}$. Controls for specificity of immunohistochemistry were performed by: (1) omitting any essential step of the immunoreaction or (2) substitution of the anti-rabbit IgG with appropriate dilution of normal (non-immune) goat serum. Positive controls of the techniques were performed in rabbit tissue (data not shown). Analysis of the samples was independently performed by 2 experienced observers (A.B-F. and I.W.). No numerical analysis was performed, because the study was purely descriptive.

\section{Results}

Binding of ATGs to endothelial cells were demonstrated in vivo in our animal experiments at $4 \mathrm{~h}$ after reperfusion, as well as in the clinical biopsies after induction of immunosuppression with ATGs in heart transplant patients.

\section{Animal biopsies}

Positive immunohistochemical staining demonstrated binding of ATGs to endothelial cells after 4-h reperfusion with human blood treated with thymoglobulin. This binding was shown in all slides. The polyclonal antibodies were preferentially located in venous endothelial cells, especially in post-capillary veins. Figure 1 shows a representative image of ATGs binding to ECs. Figure $3 \mathrm{~A}$ shows an absence of positive reaction in animals not treated with ATG.

\section{Human biopsies}

Morphological differences were typical within the recipient (diseased) and donor cardiac biopsies. H/E staining of diseased muscle presented necrotic muscle fibers, loss of normal muscular tissue architecture, and diffuse interstitial fibrosis in all the biopsies studied. Biopsies from donor hearts showed normal cardiac morphology and no evidence of spontaneous inflammation or increased fibrosis.

The expression of anti-rabbit-lgG in myocardium was evaluated by immunohistochemistry. There was positive staining after induction with ATGs in endothelial cells, both in the recipient heart (at least $3 \mathrm{~h}$ after induction; Figure 2A, 2B) and in the donor heart (at least $5 \mathrm{~h}$ after induction; Figure $2 \mathrm{C}$ ). Localization was preferentially in venous vessels. Positive reaction for immune cells, especially lymphocytes and polymorphonuclear cells, was observed, especially in arterial vessels. Cardiac biopsies obtained 7 days after heart transplantation showed positive staining for anti-rabbit-lgG, mostly on intraluminal immune cells, showing scarce positive immunostaining on endothelial cells. This fact suggests that circulating blood is able to wash out ATGs previously bound to ECs (Figure 2D). The positive controls of the technique performed in rabbit muscle and connective tissue (data not shown) revealed an intense positive reaction, confirming the positive reaction in human tissue. Absence of staining was demonstrated in patients with end-stage heart failure undergoing VAD implantation and not treated with ATG (Figure 3B).

\section{Discussion}

Although rabbit ATGs have been used in thousands of solid organ transplants for more than 30 years after licensing, the immunological properties of these drugs are not fully understood. In fact, growing knowledge of the complex immunological activity of rATGs allows new clinical applications in solid organ transplantation and hematological diseases, and requires new research in other therapeutic fields [4].

The main mechanism of action of rATGs has been traditionally related to T-cell and NK-cell depletion through complement activation and induction of apoptosis through CD95 [1]. Furthermore, depletion of activated $\mathrm{CD} 3+, \mathrm{CD} 4+$, and $\mathrm{CD} 8+$ followed by reconstitution of CD4+ from naïve CD4 T-cells has been recently described, and is a pivotal role of these antibodies $[13,14]$. This mechanism of action is responsible for the clinical activity of ATGs in induction of immunosuppression, prevention and treatment of acute rejection, and treatment of graft-versus-host disease (GvHD).

However, there is growing evidence that the immunological action of rabbit ATGs is not only dependent on T-cell depletion, but is also related to other mechanisms of action, such as modulation of adhesion molecules and chemokine receptors, as well as regulation of different immune cells, such as B- and NK-cells, 
dendritic cells, and regulatory T-lymphocytes [3]. Regulation of adhesion molecules, such as ICAM or PECAM, and cell-trafficking molecules, such as CXCR4 or CCR5, through ATGs is of special importance, as these molecules expressed on the surface of leukocytes and endothelial cells play a key role in ischemia-reperfusion injury (IRI) and acute rejection [15-17]. Our research group has provided experimental evidence of a reduction of IRI and tissue infiltration and reduction of tissue apoptosis after treatment with ATGs in a primate model $[3,11]$. Furthermore, we demonstrated modulation of adhesion and inflammatory molecules on the endothelial surface, which could be related to decreased leukocyte-endothelial interactions and improved microcirculation after ischemia $[12,15]$.

The affinity of rabbit ATGs for endothelial surface molecules has been sufficiently demonstrated $[15,16,18]$. We have demonstrated a dose-dependent binding of ATGs to HUVECs and adult endothelial cells by means of flow cytometry in previous studies $[5,10]$. We also showed a decrease of trans-endothelial migration of leukocytes after treatment of ECs with thymoglobulin [19]. In the present study we demonstrated the binding of ATGs to endothelial cells in an experimental model and in clinical practice. Our results provide an explanation for the decrease of leukocyte-endothelial interactions, postulated in previous studies [12,15] as coating of endothelial cells may reduce the recognition of antigens presented on the endothelial surface, preventing adhesion of circulating leukocytes and trans-endothelial migration of leukocytes in vivo. Interestingly, the expression of ATGs in ECs was reduced after 7 days. This fact might be explained through a washout phenomenon of the circulating blood, and would provide a rationale for repeated low-dose administration of ATGs at intervals.

Other advantages can be proposed as hypotheses for a beneficial effect of ATGs in the transplant setting regarding endothelial binding. Brokhof et al. demonstrated a lower incidence of

\section{References:}

1. Beiras-Fernandez A, Thein E, Hammer C: Induction of immunosuppression with polyclonal antithymocyte globulins: an overview. Exp Clin Transplant, 2003; 1: 79-84

2. Beiras-Fernandez A, Thein E, Chappel $D$ et al: Polyclonal anti-thymocyte globulins influence apoptosis in reperfused tissues after ischaemia in a non-human primate model. Transpl Int, 2004; 17(8): 453-57

3. Mohty M: Mechanisms of action of antithymocyte globulin: T-cell depletion and beyond. Leukemia, 2007; 21: 1387-94

4. Mohty M, Bacigalupo A, Saliba F et al: New directions for rabbit antithymocyte globulin (Thymoglobulin ${ }^{\circledR}$ ) in solid organ transplants, stem cell transplants and autoimmunity. Drugs, 2014; 74: 1605-34

5. Kanzler I, Seitz-Merwald I, Schleger S et al: In vitro effects of ATG-Fresenius on immune cell adhesion. Transplant Proc, 2013; 45(5): 1846-49

6. Cherkassky L, Lanning M, Lalli PN et al: Evaluation of alloreactivity in kidney transplant recipients treated with antithymocyte globulin versus IL-2 receptor blocker. Am J Transplant, 2011; 11: 1388-96

7. Aliabadi A, Grömmer M, Cochrane A et al: Induction therapy in heart trans plantation: Where are we now? Transplant International. 2013; 26: 684-695. de novo DSA and antibody-mediated rejection after induction of immunosuppression with rATGs in comparison to Basiliximab after kidney transplantation [8]. Binding of ATGs to endothelial cells in the first days after transplantation might reduce the function of ECs as "antigen presenting cells", thus decreasing the expression of antigens leading to production of antibodies through B-cells.

Another possible beneficial effect of rATGs binding to ECs is the protection against delayed graft function (DGF). It has been proposed in experimental studies that ATGs can reduce the incidence of DGF through reduction of activated T-cells and reduction of the leukocyte endothelial interactions [12,15]. Binding of ATGs to ECs could explain the reduction of early infiltration of the graft's parenchyma, thus providing protection against malfunction. However, clinical data proving this hypothesis are lacking. However, a multicenter clinical trial in France has been recently initiated to investigate the beneficial effect of ATGs in DGF [20].

The main objective of our study was to demonstrate the binding of rabbit ATGs to endothelial cells in vivo. We cannot provide a mechanistic explanation, although several previous studies showed interaction between surface antigens of ECs and ATGs. Another disadvantage of our study is that our analysis was performed with only 1 application of ATG preoperatively, thus possibly reducing the efficacy of the drug due to under-dosage.

\section{Conclusions}

Our results show that ATGs are able to bind to endothelial cells in an experimental model and in clinical practice, thus explaining different mechanisms of action of these drugs. Our results could support the idea of preconditioning strategies with ATGs in solid organ transplantation to prevent endothelial damage.

8. Brokhof MM, Sollinger HW, Hager DR et al: Antithymocyte globulin is associated with a lower incidence of de novo donor-specific antibodies in moderately sensitized renal transplant recipients. Transplantation, 2014; 97(6): 612-17

9. Popow I, Leitner J, Grabmeier-Pfistershammer $\mathrm{K}$ et al: A comprehensive and quantitative analysis of the major specificities in rabbit antithymocyte globulin preparations. Am J Transplant, 2013; 13: 3103-13

10. Walther S, Beiras-Fernandez A, Csapo C et al: Influence of polyclonal ATGs on the expression of adhesion molecules of isolated human umbilical vein endothelial cells. Transplant Proc, 2010; 42(5): 1931-34

11. Beiras-Fernandez A, Chappell D, Hammer C, Thein E: Influence of polyclonal anti-thymocyte globulins upon ischemia-reperfusion injury in a non-human primate model. Transpl Immunol, 2006; 15(4): 273-79

12. Chappell D, Beiras-Fernandez A, Hammer C, Thein E: In vivo visualisation of the effect of polyclonal antithymocyte globulins on the microcirculation after ischaemia/reperfusion in a primate model. Transplantation, 2006; 81(4): 552-58 
13. Lindemans CA, Chiesa R, Amrolia PJ et al: Impact of thymoglobulin prior to pediatric unrelated umbilical cord blood transplantation on immune reconstitution and clinical outcome. Blood, 2014; 123: 126-32

14. Morelon E, Lefrancois N, Besson C et al: Preferential increase in memory and regulatory subsets during T-lymphocyte immune reconstitution after thymoglobulin induction therapy with maintenance sirolimus vs. cyclosporine. Transpl Immunol, 2010; 23: 53-58

15. Beiras-Fernandez A, Chappell D, Hammer C et al: Impact of polyclonal anti-thymocyte globulins on the expression of adhesion and inflammation molecules after ischemia-reperfusion injury. Transpl Immunol, 2009; 20(4): 224-28

16. Michallet $M C$, Preville $X$, Flacher $M$ et al: Functional antibodies to leukocyte adhesion molecules in antithymocyte globulins. Transplantation, 2003; 75(5): 657-62
17. Merion RM, Howell T, Bromberg JS: Partial T-cell activation and anergy in duction by polyclonal antithymocyte globulin. Transplantation, 1998; 65(11): 1481-89

18. Préville $X$, Flacher $M$, LeMauff $B$ et al: Mechanisms involved in antithymocyte globulin immunosuppressive activity in a nonhuman primate model. Transplantation, 2001; 71(3): 460-68

19. Werner I, Bogert NV, Stock UA et al: Dose/time-dependent modulation of the endothelial function through induction agents: non-depleting versus depleting agents. Transplant Proc, 2014; 46(9): 2953-56

20. Chapal M, Foucher Y, Marguerite $M$ et al: PREventing Delayed Graft Function by Driving Immunosuppressive InduCtion Treatment (PREDICT-DGF): Study protocol for a randomized controlled trial. Trials, 2015; 16: 282 